\title{
High-fat diet feeding alters metabolic response to fasting/non fasting conditions. Effect on caveolin expression and insulin signalling
}

\author{
Ana Gómez-Ruiz ${ }^{2}$ Fermín I Milagro², Javier Campión², J Alfredo Martínez² and Carlos de Miguel ${ }^{* *}$
}

\begin{abstract}
Background: The effect of food intake on caveolin expression in relation to insulin signalling was studied in skeletal muscle and adipocytes from retroperitoneal (RP) and subcutaneous (SC) adipose tissue, comparing fasted (F) to not fasted (NF) rats that had been fed a control or high-fat (HF) diet for 72 days.

Methods: Serum glucose was analysed enzymatically and insulin and leptin by ELISA. Caveolins and insulin signalling intermediaries (IR, IRS-1 and 2 and GLUT4) were determined by RT-PCR and western blotting. Caveolin and IR phosphorylation was measured by immunoprecipitation. Data were analysed with Mann-Whitney $U$ test.

Results: High-fat fed animals showed metabolic alterations and developed obesity and insulin resistance. In skeletal muscle, food intake (NF) induced activation of IR and increased expression of IRS-2 in control animals with normal metabolic response. HF animals became overweight, hyperglycaemic, hyperinsulinemic, hyperleptinemic and showed insulin resistance. In skeletal muscle of these animals, food intake (NF) also induced IRS-2 expression together with IR, although this was not active. Caveolin 3 expression in this tissue was increased by food intake (NF) in animals fed either diet. In RP adipocytes of control animals, food intake (NF) decreased IR and IRS-2 expression but increased that of GLUT4. A similar but less intense response was found in SC adipocytes. Food intake (NF) did not change caveolin expression in RP adipocytes with either diet, but in SC adipocytes of HF animals a reduction was observed. Food intake (NF) decreased caveolin-1 phosphorylation in RP but increased it in SC adipocytes of control animals, whereas it increased caveolin-2 phosphorylation in both types of adipocytes independently of the diet.
\end{abstract}

Conclusions: Animals fed a control-diet show a normal response to food intake (NF), with activation of the insulin signalling pathway but without appreciable changes in caveolin expression, except a small increase of caveolin-3 in muscle. Animals fed a high-fat diet develop metabolic changes that result in insulin signalling impairment. In these animals, caveolin expression in muscle and adipocytes seems to be regulated independently of insulin signalling.

\section{Background}

Obesity is a complex multifactorial condition that results from a combination of environmental (such as imbalanced eating habits and sedentary lifestyle) and neuroendocrine factors, coupled to a genetic predisposition [1]. Different genes have been related to obesity development, such as the three major isoforms of caveolin, Cav-1, Cav-2 and Cav-3 (18-24 kDa) [2]. Cav-1 is most abundantly expressed in terminally differentiated

\footnotetext{
* Correspondence: cdmiguel@unav.es

'Department of Biochemistry and Molecular Biology, University of Navarra, Pamplona, Spain

Full list of author information is available at the end of the article
}

cells such as fibroblasts, epithelial and endothelial cells and adipocytes, where it is responsible for caveolae formation [3]. Cav-2 is coexpressed with Cav-1, while Cav3 is the specific isoform of muscle tissue, although it has also been found in astrocytes and chondrocytes $[4,5]$. These proteins are the main structural components of caveolae and interact with signalling molecules through a characteristic scaffolding domain [6]. Enhanced cellular signalling within caveolae is facilitated due to the target-rich environment formed by the clustering of receptors and signalling molecules in the proximity of these membrane structures, thereby permitting a better controlled and more efficient signal

\section{Ciomed Central}


transduction [7]. Insulin receptor (IR) is among those that can be located in caveolae and in fact, several studies have shown that, in adipocytes, Cav-1 is an important regulatory element stimulating IR signalling and linking insulin action to glucose uptake [8].

In obesity-related disorders, such as insulin resistance and type 2 diabetes, insulin signalling becomes altered, while adipose tissue develops chronic inflammation and hypoxia, conditions that affect gene expression through the associated oxidative stress and reactive oxygen species (ROS) production [9]. In regard to this, caveolin expression is highly dependent on proinflammatory factors such as TNF-alpha [10], and oxidative stress induces cellular senescence through activation of the Cav-1 promoter and upregulation of Cav-1 protein expression [11].

In addition, the two major targets of insulin action are skeletal muscle and adipose tissue [12]. White adipose tissue (WAT) serves as the main site for energy storage in the form of triglycerides, but also contributes to systemic glucose and lipid regulation acting as an endocrine organ [13]. The principal site of glucose uptake under insulin-stimulated conditions is skeletal muscle, being considered a primary site for insulin resistance [14]. An impairment of the initial steps in insulin signalling transduction pathways could contribute to the deficiency in insulin-stimulated glucose uptake in skeletal muscle, thus resulting in insulin resistance. In fact, different mechanisms have been described in relation to lipid-induced muscle insulin resistance, including acute free fatty acid elevation and prolonged lipid accumulation in muscle [15].

In previous studies, our group has demonstrated that caveolins are time-dependently regulated by dietinduced obesity [16]. In a late phase, after a prolonged time feeding on a high-fat diet, insulin resistance becomes apparent, accompanied by an impairment of Cav-3 and Cav-1 in skeletal muscle [17], whereas in adipocytes, Cav-1 and Cav-2 expression are increased, together with an inhibition of insulin signalling intermediaries [18].

The purpose of the present study is to deepen into the understanding of caveolin regulation in conditions of diet-induced obesity and insulin resistance, and their relation to insulin signalling in skeletal muscle and adipocytes. Given that fasting, the usual condition in animal studies before isolating samples, downregulates insulin secretion and signalling and therefore would influence caveolin physiology, we have compared for the first time caveolin regulation between fasted (F) and fed (non-fasted, NF) animals. We have analysed the response of skeletal muscle and adipocytes isolated from visceral and subcutaneous locations of lean ( $\mathrm{C}$, control) and high-fat (HF) diet-induced obese, insulin-resistant rats.

\section{Methods}

Animals, diets and experimental design

Male Wistar rats (250-300 g) were supplied by the Applied Pharmacobiology Center (University of Navarra, Spain) and housed under controlled conditions of temperature $\left(22 \pm 2^{\circ} \mathrm{C}\right)$, relative humidity $(55 \pm 10 \%)$ and 12 hours light cycle ( 8 a.m. to 8 p.m.). Sixteen animals were assigned to two different dietary groups for 72 days. The control group $(C, n=8)$ was fed standard laboratory pelleted diet (Harlam Iberica, Barcelona, Spain), providing $350 \mathrm{kcal} / 100 \mathrm{~g}$, with about $10 \%$ of the energy as fat, $73 \%$ as carbohydrates and $17 \%$ as protein. A second group $(\mathrm{n}=8)$ was fed a fat-rich cafeteria diet $(\mathrm{HF})$, providing $430 \mathrm{kcal} / 100 \mathrm{~g}$, with about $59 \%$ of the energy as fat, $32 \%$ as carbohydrates and $9 \%$ as protein, which was prepared with pate, chips, chocolate, bacon, biscuits and chow in a proportion of 2:1:1:1:1:1, as previously reported [19]. Animals had ad libitum access to food and water during the treatment. Weight gain and food/water intake were measured three times per week. Twelve hours before sacrifice 4 animals of each group were fasted (F) by food withdrawal (C-F and HF-F), whereas the other 4 were not fasted (NF), having free access to food (C-NF and HF-NF). After sacrifice by decapitation, truncal blood was collected and the gastrocnemius muscle and the retroperitoneal (RP) and subcutaneous (SC) white adipose fat pads were carefully excised and weighed. Muscle samples were immediately frozen in liquid nitrogen and stored at $-80^{\circ} \mathrm{C}$ until use. RP and SC adipose samples were immediately processed for adipocyte isolation. All the procedures were performed according to national guidelines and under care of the Animal Care and Use Committee at the University of Navarra.

\section{Adipocyte isolation}

$\mathrm{RP}$ and $\mathrm{SC}$ white fat samples were minced using fine scissors during 1 minute for RP and 2 minutes for SC [20]. Grinded tissue was digested at $37^{\circ} \mathrm{C}$ in KRBA buffer (Krebs-Ringer bicarbonate) containing collagenase type II $(1.25 \mathrm{~g} / \mathrm{ml})$, during 30 minutes for RP and 40-60 minutes for SC, the digestion was stopped adding $24 \mathrm{ml}$ of KRBA buffer. Adipocytes were separated from stromal cells by passing through mesh tissue, and washed three times at $37^{\circ} \mathrm{C}$ with $15 \mathrm{ml}$ of KRBA buffer during 5-10 minutes before freezing in liquid nitrogen to keep them stored al $-80^{\circ} \mathrm{C}$ until use.

\section{Analysis of blood samples}

Serum glucose was analyzed using an Autoanalyzer (COBAS Roche Diagnostic, Basel, Switzerland) by 
enzymatic routine procedures. Serum insulin (Mercodia A-B, Uppsala, Sweden) and leptin (Rat Leptin ELISA Kit, Linco Research, St Charles MO, USA) levels were measured by ELISA. HOMA index (Homeostasis Model Assessment) was calculated as fasting insulin $(\mu \mathrm{U} / \mathrm{ml}) \times$ fasting glucose $(\mathrm{mmol} / \mathrm{L}) / 22.5$.

\section{Reverse Transcripcion (RT)-PCR}

Total RNA was extracted from frozen skeletal muscle and RP and SC adipocytes, with Trizol (Invitrogen, Carlsbad CA, USA). Contaminating genomic DNA was removed by treatment with DNase (DNA-free ${ }^{\mathrm{TM}}$ kit, Applied Biosystems, Austin TX, USA) and purified total RNA was used as a template to generate first strand cDNA synthesis using M-MLV reverse transcriptase (Invitrogen, Carlsbad CA, USA) and random hexamers (Applied Biosystems, Austin TX, USA) as primers. Quantitative real-time PCR was performed as described by the provider (Applied Biosystems, Austin TX, USA) using an ABI-PRISM 7300 HT Sequence Detection System and Taqman probes for rat Cav-1, Cav-2, Cav-3, GLUT4, IR, IRS-1 and IRS-2. GAPDH was used as internal control for RT-PCR efficiency and subsequent normalization. The results were calculated by the $2^{-\Delta \Delta \mathrm{Ct}}$ method [21].

\section{Western Blot Analyses}

Skeletal muscle samples were homogenized with HES lysis buffer containing $20 \mathrm{mM}$ HEPES, 5 mM EDTA, 250 $\mathrm{mM}$ sucrose, and $1 \mathrm{X}$ protease inhibitor mix (SigmaAldrich, Madrid, Spain) pH 7.5. RP and SC adipocytes samples were homogenized with the same buffer by pippeting. Cell lysates were cleared by centrifugation $(12,000$ g) at $4^{\circ} \mathrm{C}$ for 45 minutes and supernatants were stored at $-80^{\circ} \mathrm{C}$. Protein content was determined with bincinchoninic Acid Kit (Sigma-Aldrich Madrid, Spain). For skeletal muscle, 10 to $15 \mu \mathrm{g}$, and for adipocytes, 20 to $50 \mu \mathrm{g}$ protein were separated by SDS-PAGE gel electrophoresis at 130-150 V for 45 minutes, and electro-transferred to nitrocellulose membranes (Schleicher \& Schuell, Amersham Biosciences, Piscataway NJ, USA) at $300 \mathrm{~mA}$ for one hour. Non-specific binding sites were blocked for $2 \mathrm{~h}$ at room temperature with Tris-base buffer containing $0.1 \%$ Tween 20 and $4 \%$ non-fat milk except for Cav-2 membranes that were blocked with Tris-base buffer containing only $10 \%$ Tween 20 . After blocking, membranes were incubated overnight with specific primary antibodies at suitable dilutions. Secondary HRP-conjugated antibodies were added for one hour and the signal developed with the Super Signal West Pico Chemiluminescence Substrate (Pierce Biotechnology, Inc., Rockford IL, USA). Membranes were exposed to Hyperfilm ECL blotting (Amersham Biosciencies, Piscataway NJ, USA). When necessary, blots were stripped by immersion in Red-Blot Plus solution 10X (Millipore, Millipore, Billerica
MA, USA) and reprobed with different antibodies, using the same procedure described above. Protein bands were quantified by Quantity One data analyzer software 4.6.3 (Bio-Rad, Muenchen, Germany) and normalized to the $\beta$ actin signal as internal control. Cav-1 (1/50,000), Cav-2 $(1 / 20,000)$ for skeletal muscle and $(1 / 250)$ for RP and SC adipocytes, and Cav-3 $(1 / 10,000)$ antibodies were supplied by Santa Cruz Biotechnology Inc., (Santa Cruz CA, USA). Insulin receptor IR $(1 / 10,000)$ and Phosphotyrosine $(1 / 10,000)$ for skeletal muscle and $(1 / 5,000)$ for RP and $\mathrm{SC}$ adipocytes, antibodies were obtained from Cell Signalling Technology, (Danvers MA, USA) and $\beta$-actin $(1 / 10,000)$ antibody was purchased from Sigma-Aldrich (St. Louis MO, USA). The secondary antibodies were anti-mouse $(1 / 10,000)$ from Amersham Biosciences-GE Healthcare (Piscataway, NJ, USA), anti-rabbit $(1 / 10,000)$, from Sigma-Aldrich (St. Louis MO, USA) and anti-goat $(1 / 10,000)$, from Santa Cruz Biotechnology Inc. (Santa Cruz CA, USA).

\section{Inmunoprecipitation}

Samples containing 250-500 $\mu$ g of total protein were incubated with antibodies against Cav-1 (1/100 v/w), Cav-2 (1/100 v/w), Cav-3 (1/100 v/w) and IR (1/50 v/w) overnight at $4{ }^{\circ} \mathrm{C}$ on a rotating device, followed by addition of Protein A-G PLUS Agarose (Santa Cruz Biotechnology Inc., Santa Cruz CA, USA) for 2 hours in the same conditions. Immunoprecipitation complexes were washed three times with $500 \mu \mathrm{l}$ of HES lysis buffer containing $20 \mathrm{mM}$ HEPES, $5 \mathrm{mM}$ EDTA, $250 \mathrm{mM}$ sucrose and $1 \mathrm{X}$ protease inhibitor (Sigma-Aldrich Madrid, Spain) $\mathrm{pH}=7.5$ for skeletal muscle samples, and with $500 \mu \mathrm{l}$ of lysis buffer $(0.25 \%$ sodium deoxicholate, 50 $\mathrm{mM}$ Tris- $\mathrm{HCl} \mathrm{pH}$ 7.5, $150 \mathrm{mM} \mathrm{NaCl}, 1 \%$ Triton X-100, $5 \mathrm{mM}$ EDTA, $2 \mathrm{mM} \mathrm{NaF}, 2 \mathrm{mM}$ sodium ortovanadate, $6 \mathrm{mM}$ octil-glucoxide and $1 \mathrm{X}$ protease inhibitor mix (Sigma-Aldrich, Madrid Spain) for adipocyte samples. Supernatants were discarded and pellets resuspended in $20 \mu \mathrm{l}$ Laemmli sample buffer $1 \mathrm{X}$. After $7 \mathrm{~min}$ boiling, samples were centrifuged and analyzed by Western blotting with the corresponding antibodies as described above. Phosphorylated IR and caveolin bands were normalized with total IR or caveolin as reference.

\section{Data analysis}

The results were expressed as the mean \pm S.E.M. Data were compared using Mann-Whitney $U$ test. All analyses were performed using SPSS version 15.0 for Windows.

\section{Results}

Body, tissue weight and blood determinations

As previously reported using the same dietary model [17], body and adipose depot weights of the animals fed 
on the HF-diet were significantly higher than those fed on the control diet. However, no differences were found between the fasted (F) and non-fasted (NF) groups of animals (Table 1). Only gastrocnemius muscle weight decreased by fasting independently of the diet (Table 1), probably due to glycogen depletion and protein mobilization from muscle tissue [22].

Animals grown on the control diet responded normally to feeding (NF) and showed increased levels of glucose, insulin and leptin as a consequence of food intake (Table 1). On the other hand, animals fed on the HF-diet were metabolically altered showing insulin resistance, hyperglycemia, hyperinsulinemia and hyperleptinemia. Therefore, the circulating levels of glucose, insulin and leptin were either not affected (glucose) or only slightly increased (insulin and leptin) by continuous feeding (NF) (Table 1). These results suggests a preliminary state of insulin resistance in these animals, as indicated by their significantly higher $(\mathrm{p}<0.01)$ HOMA index: (C) $2.99 \pm 0.68$, (HF) $14.07 \pm 3.99$.

\section{mRNA expression of insulin pathway intermediaries}

In skeletal muscle, control-fed animals showed an increase in IRS-2 expression when not fasted (NF), which was maintained in HF-fed animals (Figure 1A). Although not significant, GLUT4 expression was also higher in NF control animals, but this difference was lower in HF-fed animals (Figure 1A). GLUT4 mRNA levels were also higher in adipocytes of NF control animals, whether they came from the RP or the SC pads, and this difference was also reduced in HF-fed animals, specially in SC adipocytes (Figure 1B, C). On the contrary, in RP adipocytes, IR and IRS-2 expression were slightly down-regulated in NF animals independently of the diet (Figure 1B). In SC adipocytes a similar trend was observed, but this difference only reached marginal significance for IR in control-fed animals (Figure 1C). As expected, muscle IR-phosphorylation was higher in the control-diet fed NF animals as compared to the fasted ones. However, this activation decreased in the NF animals fed the HF diet (Figure 2C).

\section{Caveolin expression and activation in skeletal muscle}

Neither expression of Cav-1 and Cav-2 nor phosphorylation level of Cav-1 were affected by the feeding status with either diet (Figure 2). Only Cav-3 expression seemed to be higher in NF animals, showing increased mRNA levels in the HF-fed group and higher protein levels in the control-fed group (Figure 2).

\section{Caveolin expression and activation in adipocytes from retroperitoneal white adipose tissue}

Caveolin expression did not change in NF animals either at mRNA or protein level (Figure 3A, B). However, significant variations in caveolin activation were observed when their state of phosphorylation was measured. Cav1 phosphorylation was reduced in NF animals fed the control diet but increased in animals fed the HF diet (Figure 3C). On the other hand, Cav-2 showed enhanced phosphorylation in NF animals independently of the diet (Figure 3C).

\section{Caveolin expression and activation in adipocytes from subcutaneous white adipose tissue}

In contrast to adipocytes from the RP pad, some changes were observed in caveolin expression in SC adipocytes. Thus, NF animals fed the HF diet showed decreased levels of Cav-1 mRNA and of Cav-2 protein. A reduction of Cav-1 protein and Cav-2 mRNA levels were also observed in the same groups of animals, but did not reached statistical significance (Figure 4A, B). Interestingly, Cav-2 phosphorylation was increased in NF animals independently of the diet (Figure 4C), following the same pattern as observed in RP adipocytes. On the other hand, Cav-1, whose phosphorylation increased only in NF control-fed animals (Figure 4C), seemed to follow the opposite pattern to that observed in RP adipocytes.

Table 1 Body and tissue weights and metabolic determinations in the four experimental groups

\begin{tabular}{|c|c|c|c|c|}
\hline & \multicolumn{2}{|c|}{ C } & \multicolumn{2}{|c|}{$\mathrm{HF}$} \\
\hline & $F$ & $\mathrm{NF}$ & $\mathrm{F}$ & NF \\
\hline Body Weight (g) & $424 \pm 7$ & $431 \pm 23$ & $508 \pm 33^{\#}$ & $538 \pm 18^{t}$ \\
\hline Skeletal Muscle (g) & $2.01 \pm 0.33$ & $2.51 \pm 0.06^{*}$ & $1.93 \pm 0.04$ & $2.64 \pm 0.10^{*}$ \\
\hline Retroperitoneal fat (g) & $7.99 \pm 1.30$ & $9.90 \pm 1.70$ & $20.49 \pm 2.75^{\#}$ & $17.64 \pm 2.57^{t}$ \\
\hline Subcutaneous fat (g) & $8.15 \pm 1.54$ & $10.99 \pm 2.12$ & $17.41 \pm 2.31^{\#}$ & $20.68 \pm 6.06$ \\
\hline Glucose $(\mathrm{mg} / \mathrm{dL})$ & $104.45 \pm 4.33$ & $124 \pm 6.22^{*}$ & $125.02 \pm 5.97^{\mathrm{t}}$ & $123.3 \pm 3.47$ \\
\hline Insulin (ng/mL) & $0.45 \pm 0.09$ & $2.05 \pm 0.14^{*}$ & $1.80 \pm 0.47^{\#}$ & $2.50 \pm 0.47^{\top}$ \\
\hline Leptin $(\mathrm{ng} / \mathrm{mL})$ & $3.53 \pm 0.84$ & $18.94 \pm 4.06^{*}$ & $17.87 \pm 3.54^{\#}$ & $20.93 \pm 5.78$ \\
\hline
\end{tabular}

C: control diet fed animals. HF: high-fat diet fed animals. F: Fasted animals. NF: Non fasted animals. Data are given as means \pm SEM. Groups were compared using the Mann-Whitney $U$ test: (F vs NF), $T<0.10 ;{ }^{*} \mathrm{p}<0.05$; (C-F vs HF-F and C-NF vs HF-NF), $\mathrm{t}<0.10, \# \mathrm{p}<0.05$. 


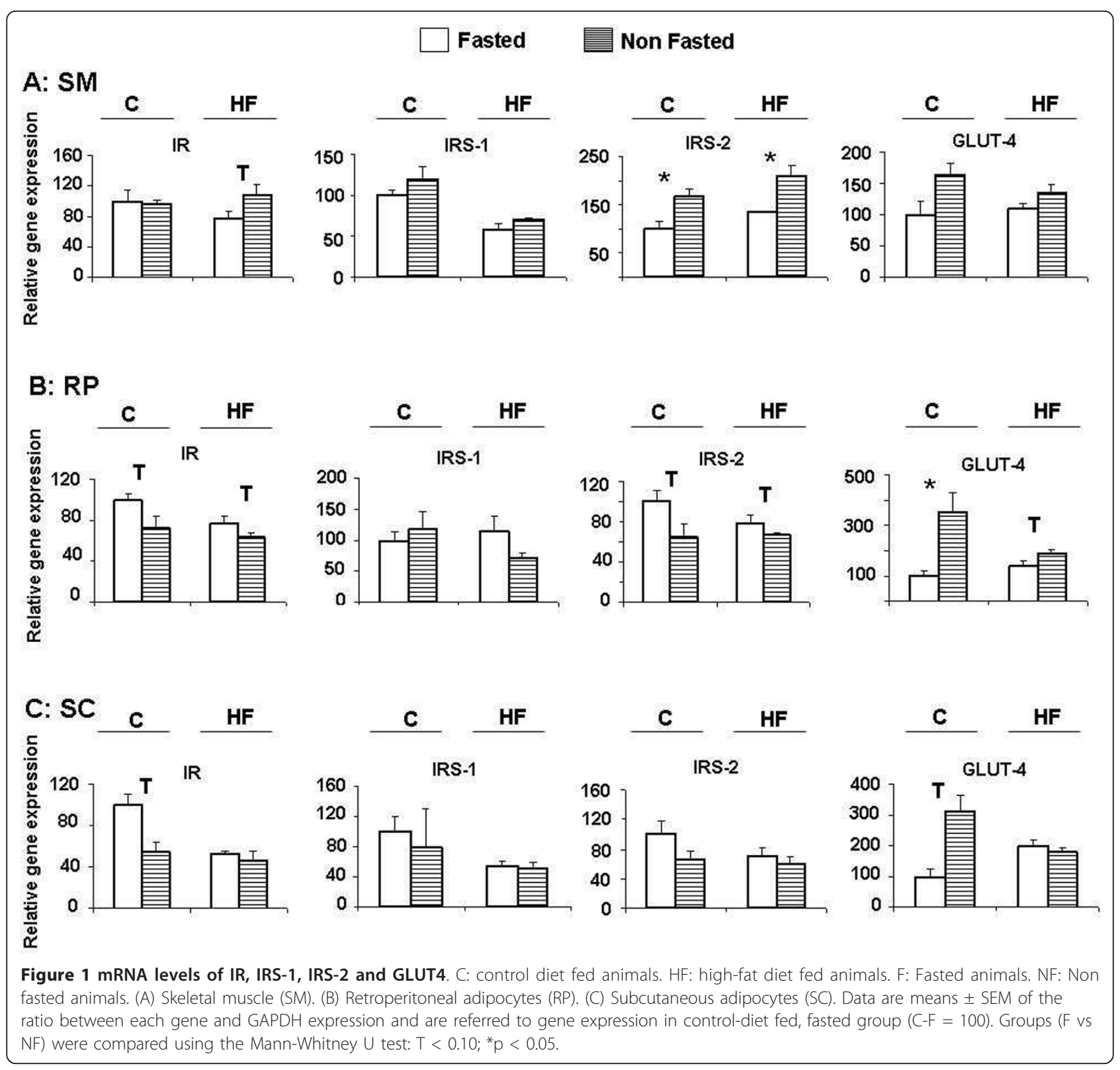

\section{Discussion}

In agreement with previous studies $[17,18]$, the current results indicate that the animals fed on a high-fat cafeteria diet for an extended period of time, besides becoming overweight, show hyperleptinemia, hyperglycemia, hyperinsulinemia and develop insulin resistance. This outcome is an indication of an altered metabolic state related to obesity development. These determinations are usually made in animals fasted overnight before being sacrificed (F), so that the differences in food intake between them during the previous hours will not influence the levels found. In the present study, a group of animals had free access to food until the moment of sacrifice and therefore were not fasted overnight (NF). These NF animals, fed a control-diet, showed the expected higher glucose and insulin levels as a consequence of food intake, which constitutes the normal metabolic response. These animals also showed increased leptin levels, explained as a satiety signal [23] (Table 1).

On the other hand, the NF animals that were fed the HF-diet experienced the mentioned insulin resistant metabolic state, which is characterized by higher levels of glucose, insulin and leptin. In this case, the continuous food availability produced smaller, although still apparent, increases in insulin and leptin levels, but no changes in circulating glucose (Table 1). 


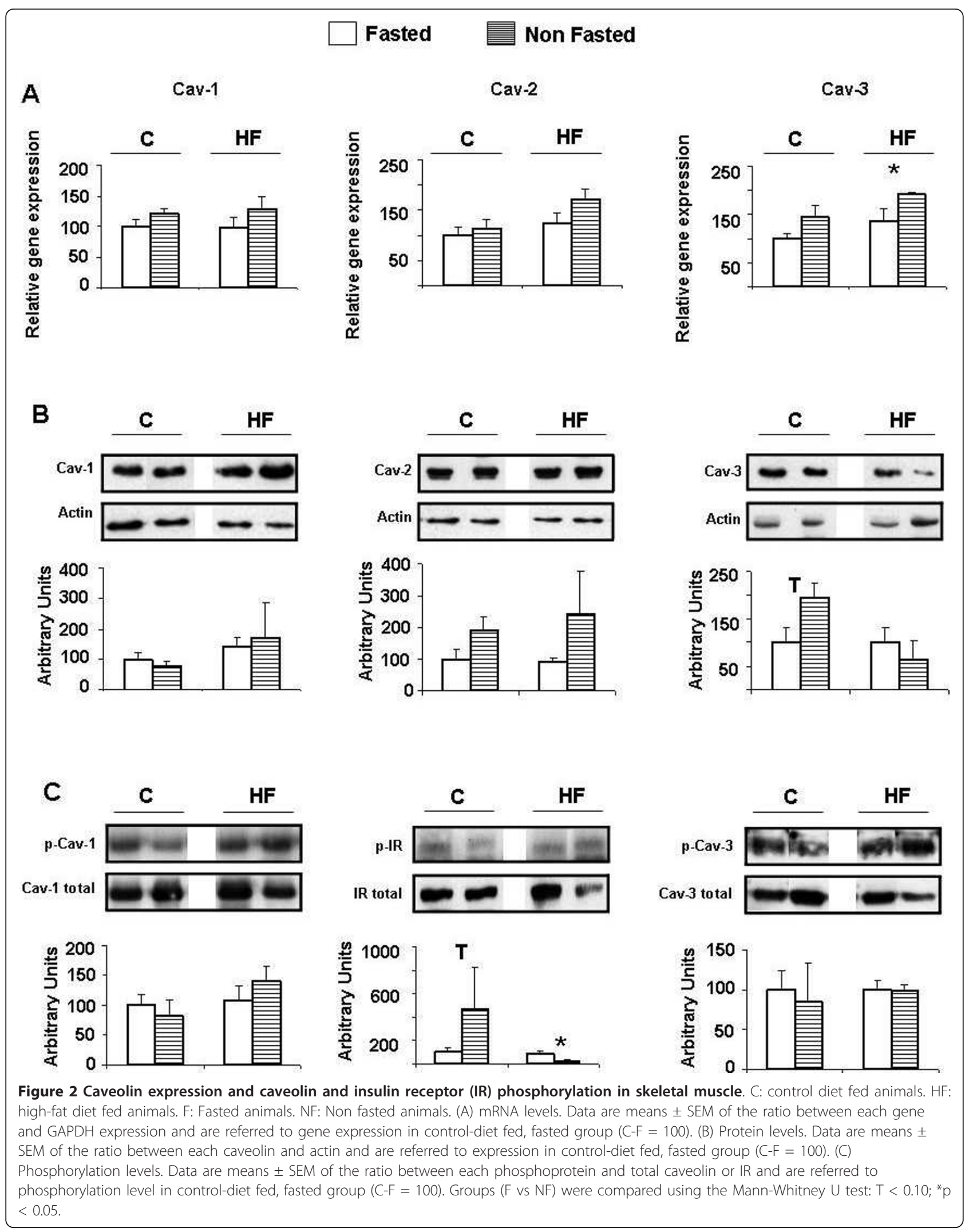




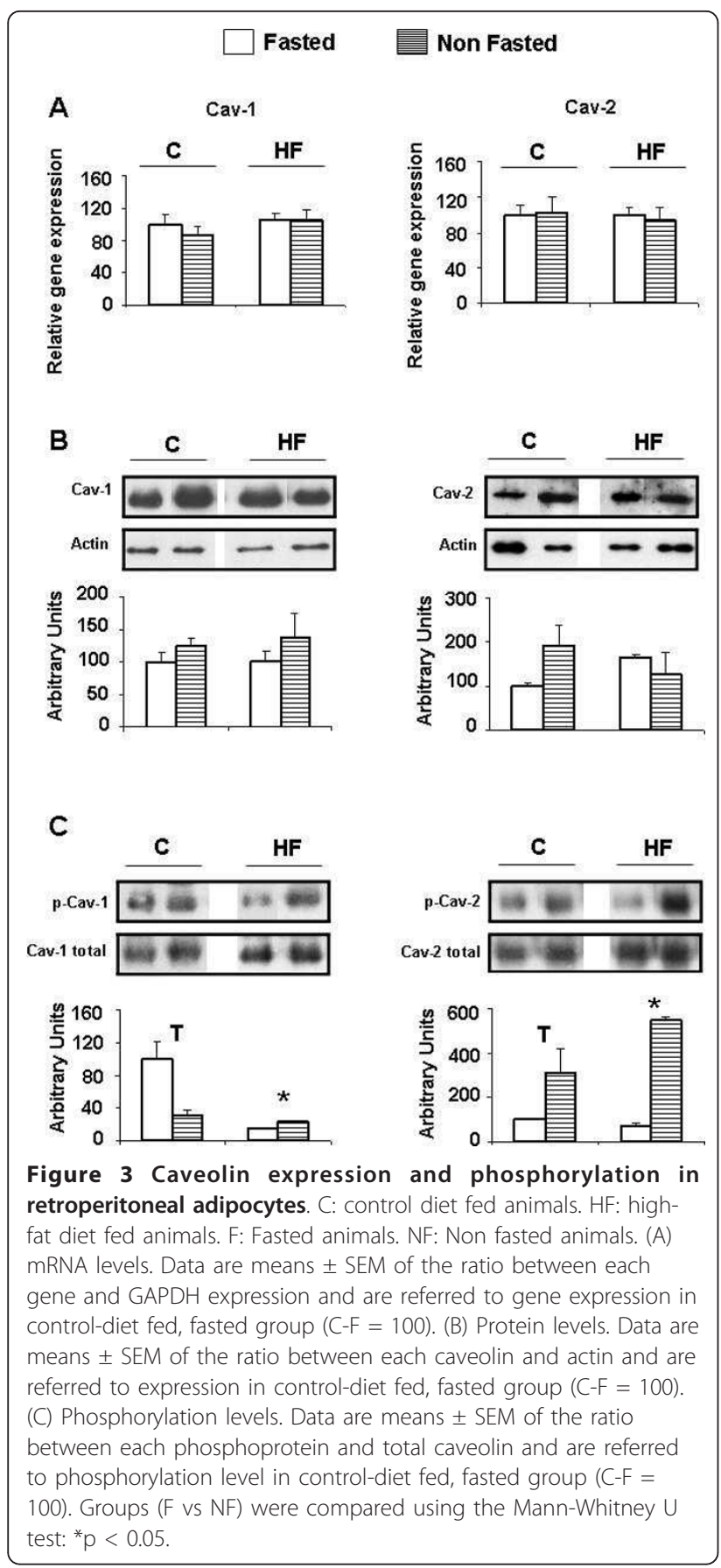

\section{Effect of non fasting in skeletal muscle}

In normal conditions, skeletal muscle is responsible for $80 \%$ of blood glucose clearance, whereas after several hours of fasting the brain becomes the main destination for circulating glucose [24]. NF control-diet fed animals had high serum glucose levels and an active insulin signalling machinery that promotes glucose entrance in muscle cells. In fact, in these animals increased IR-phosphorylation and IRS-2 expression were found (Figures 1A

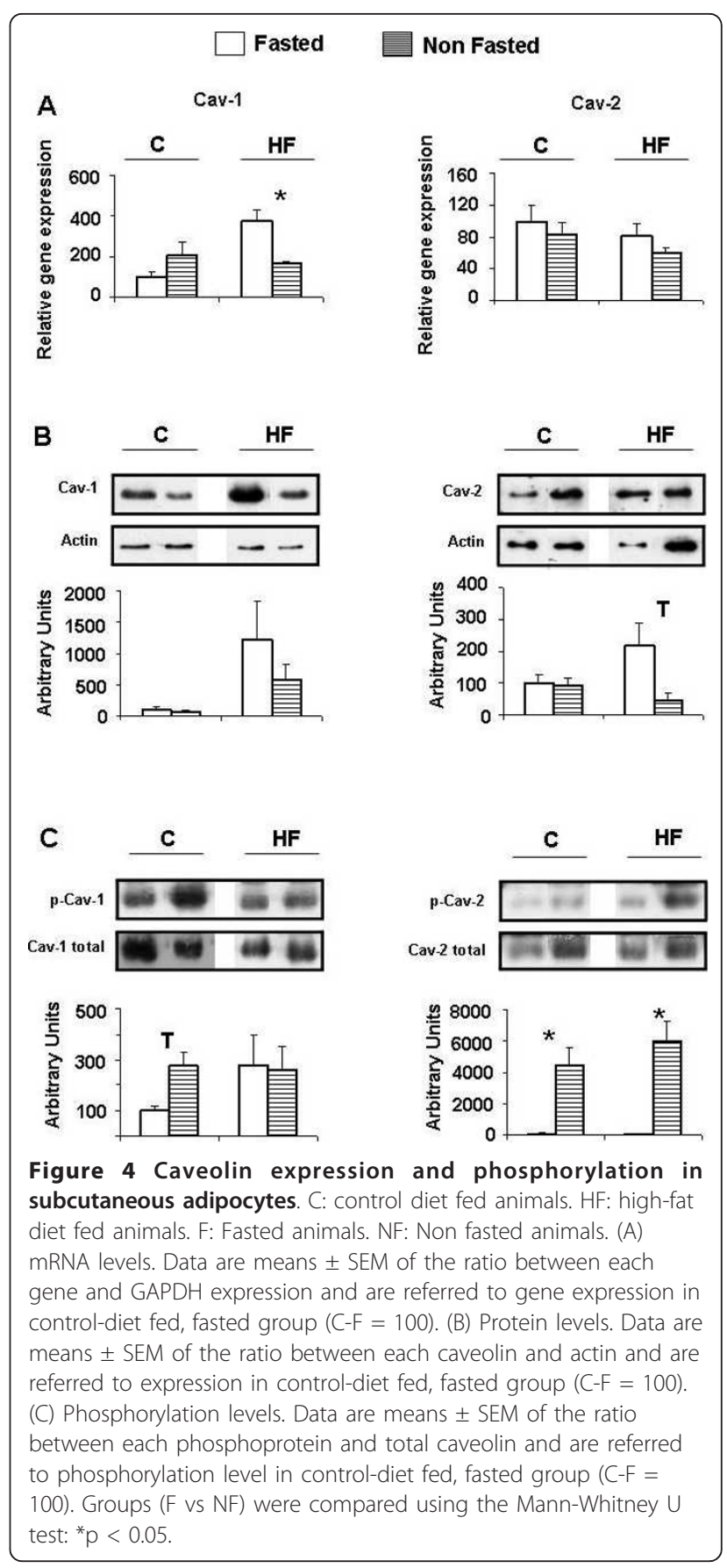

and 2C). GLUT4 expression was also slightly higher, although the difference did not reach statistical significance (Figure 1). Although IRS-1 in muscle and adipose cells is known as the main IR substrate that mediates glucose uptake through PKB/Akt activation and GLUT4 translocation to plasma membrane [25], in liver, it has been described that IRS-1 and IRS- 2 may complement each other in insulin signalling [26]. Therefore, the observed increase in IRS-2 expression (Figure 1) could 
reflect an attempt to improve insulin signalling in muscle cells. This response could be stressed in HF-diet fed animals under altered metabolic conditions, as suggested by the increased IR expression in NF individuals. Nevertheless in these animals this regulatory mechanism seems to be ineffective, since GLUT4 expression was not modified (Figure 1A) and blood glucose remained high (Table 1). As a matter of fact IR phosphorylation was diminished in NF HF-diet fed animals (Figure 2C).

Since Cav-3 is the main isoform expressed in muscle tissue, being functionally equivalent to Cav-1, it is not surprising that only Cav-3 expression seems to be affected in NF animals. Increased Cav-3 expression in normal conditions, as observed in NF control-diet fed animals, may be related to improved insulin signalling [17], although Cav-3 phosphorylation does not change (Figure 2C). In NF HF-diet fed animals Cav-3 mRNA level was higher, but this increase in expression would also be ineffective in glucose metabolism as explained before. Therefore, in agreement with previous results [17], it seems that in HF-diet fed animals, regulation of Cav-3 expression is not related to an impaired insulin signalling cascade. In regard to this, it has been reported that Cav-3 expression may be induced by oxidative stress through p38MAPK [27]. Indeed, previous studies have reported that animals fed on a high fat diet show increased oxidative stress in muscle tissue [28].

\section{Effect of non fasting in adipocytes of white adipose tissue}

Adipose tissue is considered a multifunctional organ having a critical role in metabolism and energy balance regulation, and enlargement of adipose tissue depots is the most characteristic feature of obesity [29]. However, the distribution of body fat appears to be even more important than the total amount of fat. In this way, abdominal adiposity is much more closely associated with insulin resistance, type 2 diabetes, hypertension, and dyslipidemia than subcutaneous fat mass [30]. Visceral adipose tissue is also thought to be not only metabolically more active than subcutaneous, showing higher insulin-stimulated glucose uptake [31], but also more intensely affected by obesity-related inflammation and oxidative stress [32].

In the current work, we have studied caveolin expression in relation to insulin signalling in adipocytes isolated from the visceral (RP) and SC adipose depots in animals fed a HF-diet that were fasted $(\mathrm{F})$ or not fasted (NF) before sacrifice.

In RP adipocytes it was observed that IR and IRS-2 expression were downregulated in NF control-diet fed animals whereas GLUT4 was upregulated. Analogous results were obtained in SC adipocytes, although in this case IRS-2 expression was not affected (Figure 1B,
C), and a similar observation was made in brown adipose tissue [33]. Control-diet fed animals have functional insulin signalling transmission and the GLUT4 increase may be related to insulin stimulation after feeding. In addition, Desbuqois et al. [34] reported that hyperinsulinemia can stimulate IR degradation and a decrease in IR mRNA level, and the NF control-diet fed animals, as expected, do actually exhibit higher insulin level (Table 1).

Non-fasted animals fed the HF-diet showed very similar results in RP adipocytes, but the increase in GLUT4 expression was notably less marked (Figure 1B). Since these animals have become hyperinsulinemic, blood insulin increment caused by food intake did not substantially modify the situation (Table 1), and the drop in IR or IRS-2 expression was also less pronounced (Figure 1 ). These animals have an altered insulin response that would diminish glucose uptake despite a GLUT4 expression increase. Therefore, GLUT4 expression seems to be regulated independently of the insulin signalling cascade intermediaries. In relation to this, chronic local inflammation, cell hypoxia, and the associated production of ROS and oxidative stress described in adipose tissue of obese subject [9], have been described as factors that can upregulate GLUT4 in adipocytes [35]. On the other hand, in SC adipocytes, a lack of effect was observed on the insulin intermediaries gene expression in the NF animals fed on the HF-diet (Figure 1C). This outcome is probably due to the fact that $\mathrm{SC}$ fat is metabolically less active, and therefore less sensitive to nutritional variations, than RP fat [36-38].

In RP adipocytes expression of neither of the caveolins seemed to be influenced by food intake (NF) independently of the diet (Figure 3). However, in SC adipocytes a decrease of Cav-1 mRNA and of Cav-2 protein was observed in NF HF-diet fed animals (Figure 4). In regard to this, differences in caveolin regulation have been observed between visceral and SC adipocytes from obese humans [39].

In contrast with muscle, food intake (NF) provoked changes in the phosphorylation state of caveolins in adipose tissue. Cav-1 is the isoform more directly implicated in insulin signalling in adipose tissue, therefore, under normal conditions (control-diet), increased activation of Cav-1 when glucose level rises by nutrient intake (NF), would improve insulin signal. We have observed this behaviour in the less insulin sensitive SC adipocytes (Figure 4C). However, in RP adipocytes this response might not be necessary, and indeed Cav-1 became less phosphorylated by nutrient intake (Figure $3 \mathrm{C}$ ). This effect could be related to the reduction in IR and IRS-2 expression associated to glucose level elevation produced by food intake as mentioned before. In fact, high glucose serum levels have been shown to downregulate 
Cav-1 expression in Schwann cells [40]. Cav-2 does not seem to be directly involved in insulin signalling and is considered a structural aid for functional caveolae formation. In this sense, increased Cav-2 phosphorylation by food intake in normal conditions, as observed in both RP and SC adipocytes (Figures $3 \mathrm{C}$ and $4 \mathrm{C}$ ), could be considered a mechanism to improve insulin signalling.

In the altered metabolic conditions of HF-diet fed animals, caveolin phosphorylation would not be regulated in coordination with the impaired insulin signalling cascade, but it could conceivably be more related to the increased inflammation and oxidative stress conditions associated with obesity in adipose tissue. As a matter of fact, it has been described that Cav-1 phosphorylation can be mediated by $\mathrm{p} 38 \mathrm{MAPK}$ and $\mathrm{Scr}$ through a pathway induced in response to oxidative stress [41]. In regard to this, oxidative stress response elements have been described in the promoter of caveolin-1 [42], which indeed responds to oxidative stress with an increase in its expression [43]. With respect to inflammation, LPS and different proinflamatory cytokines such as TNF-alpha and IL-1 have been shown to induce the upregulation of Cav-1 through the NF-Kappa B pathway [44]. The link between Cav-1 and these phenomena is confirmed by the observation that, the loss of Cav-1 in bone marrow-derived stromal cells from Cav-1 deficient mice, induces oxidative stress and mimics a pseudo-hypoxic state that leads to inflammation in the tumor stromal microenvironment [45]. In relation to our results it has been shown that transient hyperglycemia (i. e. nutrient intake) induces an increase in plasma IL-6, TNF-alpha, and IL-18 mediated by oxidative stress [46]. In the current work, we report that nutrient intake (NF), in a comparable situation, provokes increased phosphorylation of both caveolins in RP adipocytes and only of Cav-2 in SC cells (Figures $3 \mathrm{C}$ and $4 \mathrm{C}$ ).

These differences also strengthen the idea that both types of adipose tissue are not metabolically equivalent and have different responses in accordance with their physiological role [47].

\section{Conclusions}

Animals fed the control-diet show a normal metabolism, and food intake (NF) provokes elevation of serum glucose, insulin and leptin levels. This in turn would cause the activation of the insulin signalling pathway in muscle and adipose tissue, which will result in increased glucose uptake through the stimulation of GLUT4 activity in the cell membrane. Caveolin expression does not seem to be critically affected except in muscle, where a slight increase in Cav-3, the main isoform in this tissue, is observed.
On the other hand, animals fed the HF-diet develop a prediabetic altered metabolism in which insulin signalling is impaired. In this condition, caveolin expression in muscle and adipose tissue in response to food intake (NF) seems to be regulated independently of insulin signalling, as we have reported previously in fasted animals [18]. In summary our data clearly show that caveolins respond to nutritional changes and strengthen the role of these proteins in the regulation of energy metabolism.

\section{Acknowledgements and Funding}

The skilful technical assistance of Adela Bezunartea is gratefully acknowledged. A. Gómez was recipient of a predoctoral fellowship from "Asociación de Amigos de la Universidad de Navarra", Spain. This study was supported by grants from "Plan de Investigación de la Universidad de Navarra" (PIUNA) and Línea Especial from University of Navarra (LE/97), Spain.

\section{Author details}

'Department of Biochemistry and Molecular Biology, University of Navarra, Pamplona, Spain. ${ }^{2}$ Department of Nutrition and Food Sciences, Physiology and Toxicology, University of Navarra, Pamplona, Spain.

\section{Authors' contributions}

AG-R carried out the care and maintenance of the animals and all the experimental determinations and drafted the manuscript. FIM helped with the care and maintenance of the animals and the RT-PCR determinations, participated in the experimental design, in the revision and interpretation of the data and reviewed the manuscript. JC helped with the care and maintenance of the animals and participated in the interpretation of the data and revision of the manuscript, JAM participated in the interpretation of the data and revision of the manuscript, CdM helped with the

immunological determinations, participated in the experimental design, in the revision and interpretation of the data and drafted and reviewed the manuscript. All authors have read and approved the final manuscript.

\section{Competing interests}

The authors (AG-R; FIM; JC; JAM; CdM) report no competing interests.

Received: 22 February 2011 Accepted: 13 April 2011

Published: 13 April 2011

\section{References}

1. Campion J, Milagro F, Martinez JA: Epigenetics and obesity. Prog Mol Biol Trans/ Sci 2010, 94:291-347.

2. Williams TM, Lisanti MP: The Caveolin genes: from cell biology to medicine. Ann Med 2004, 36:584-595.

3. Scherer PE, Lisanti MP, Baldini G, Sargiacomo M, Mastick CC, Lodish HF: Induction of caveolin during adipogenesis and association of GLUT4 with caveolin-rich vesicles. J Cell Biol 1994, 127:1233-1243.

4. Schwab W, Galbiati F, Volonte D, Hempel U, Wenzel KW, Funk RH, Lisanti MP, Kasper M: Characterisation of caveolins from cartilage: expression of caveolin-1, -2 and -3 in chondrocytes and in alginate cell culture of the rat tibia. Histochem Cell Biol 1999, 112:41-49.

5. Shin T, Kim H, Jin JK, Moon C, Ahn M, Tanuma N, Matsumoto Y: Expression of caveolin-1, -2 , and -3 in the spinal cords of Lewis rats with experimental autoimmune encephalomyelitis. J Neuroimmunol 2005, 165:11-20.

6. Williams TM, Lisanti MP: The caveolin proteins. Genome Bio/ 2004, 5:214.

7. Stan RV: Structure of caveolae. Biochim Biophys Acta 2005, 1746:334-348.

8. Cohen AW, Combs TP, Scherer PE, Lisanti MP: Role of caveolin and caveolae in insulin signaling and diabetes. Am J Physiol Endocrinol Metab 2003, 285:E1151-1160.

9. Wood IS, de Heredia FP, Wang B, Trayhurn P: Cellular hypoxia and adipose tissue dysfunction in obesity. Proc Nutr Soc 2009, 68:370-377.

10. Gassmann MG, Werner S: Caveolin-1 and -2 expression is differentially regulated in cultured keratinocytes and within the regenerating epidermis of cutaneous wounds. Exp Cell Res 2000, 258:23-32. 
11. Dasari A, Bartholomew JN, Volonte D, Galbiati F: Oxidative stress induces premature senescence by stimulating caveolin-1 gene transcription through p38 mitogen-activated protein kinase/Sp1-mediated activation of two GC-rich promoter elements. Cancer Res 2006, 66:10805-10814.

12. Korenblat KM, Fabbrini E, Mohammed BS, Klein S: Liver, muscle, and adipose tissue insulin action is directly related to intrahepatic triglyceride content in obese subjects. Gastroenterology 2008, 134:1369-1375

13. Fischer-Posovszky P, Wabitsch M, Hochberg Z: Endocrinology of adipose tissue - an update. Horm Metab Res 2007, 39:314-321.

14. DeFronzo RA, Tripathy D: Skeletal muscle insulin resistance is the primary defect in type 2 diabetes. Diabetes Care 2009, 32(Suppl 2):S157-163.

15. Abdul-Ghani MA, DeFronzo RA: Pathogenesis of insulin resistance in skeletal muscle. J Biomed Biotechnol 2010, 476279.

16. Lopez IP, Milagro FI, Marti A, Moreno-Aliaga MJ, Martinez JA, De Miguel C: High-fat feeding period affects gene expression in rat white adipose tissue. Mol Cell Biochem 2005, 275:109-115.

17. Gomez-Ruiz A, de Miguel C, Campion J, Martinez JA, Milagro Fl: Timedependent regulation of muscle caveolin activation and insulin signalling in response to high-fat diet. FEBS Lett 2009, 583:3259-3264.

18. Gomez-Ruiz A, Milagro FI, Campion J, Martinez JA, de Miguel C: Caveolin expression and activation in retroperitoneal and subcutaneous adipocytes: influence of a high-fat diet. J Cell Physiol 2010, 225:206-213.

19. Garcia-Diaz D, Campion J, Milagro FI, Martinez JA: Adiposity dependent apelin gene expression: relationships with oxidative and inflammation markers. Mol Cell Biochem 2007, 305:87-94.

20. Milagro Fl, Campion J, Martinez JA: 11-Beta hydroxysteroid dehydrogenase type 2 expression in white adipose tissue is strongly correlated with adiposity. J Steroid Biochem Mol Biol 2007, 104:81-84.

21. Livak KJ, Schmittgen TD: Analysis of relative gene expression data using real-time quantitative PCR and the 2(-Delta Delta C(T)) Method. Methods 2001, 25:402-408.

22. Dohm GL, Tapscott EB, Barakat HA, Kasperek GJ: Influence of fasting on glycogen depletion in rats during exercise. J Appl Physiol 1983, 55:830-833.

23. Mars M, de Graaf C, de Groot LC, Kok FJ: Decreases in fasting leptin and insulin concentrations after acute energy restriction and subsequent compensation in food intake. Am J Clin Nutr 2005, 81:570-577.

24. Torres SH: Obesity, insulin resistance and skeletal muscle characteristics. Acta Cient Venez 1999, 50:34-41.

25. Taniguchi CM, Emanuelli B, Kahn CR: Critical nodes in signalling pathways: insights into insulin action. Nat Rev Mol Cell Biol 2006, 7:85-96.

26. Thirone AC, Huang C, Klip A: Tissue-specific roles of IRS proteins in insulin signaling and glucose transport. Trends Endocrinol Metab 2006, 17:72-78.

27. Feillet-Coudray C, Sutra T, Fouret G, Ramos J, Wrutniak-Cabello C, Cabello G, Cristol JP, Coudray C: Oxidative stress in rats fed a high-fat high-sucrose diet and preventive effect of polyphenols: Involvement of mitochondrial and NAD(P)H oxidase systems. Free Radic Biol Med 2009, 46:624-632.

28. Bonnard C, Durand A, Peyrol S, Chanseaume E, Chauvin MA, Morio B, Vidal H, Rieusset J: Mitochondrial dysfunction results from oxidative stress in the skeletal muscle of diet-induced insulin-resistant mice. J Clin Invest 2008, 118:789-800

29. Trayhurn P, Wood IS: Adipokines: inflammation and the pleiotropic role of white adipose tissue. Br J Nutr 2004, 92:347-355.

30. Hamdy O, Porramatikul S, Al-Ozairi E: Metabolic obesity: the paradox between visceral and subcutaneous fat. Curr Diabetes Rev 2006, 2:367-373.

31. Virtanen KA, Lonnroth P, Parkkola R, Peltoniemi P, Asola M, Viljanen T, Tolvanen T, Knuuti J, Ronnemaa T, Huupponen R, Nuutila P: Glucose uptake and perfusion in subcutaneous and visceral adipose tissue during insulin stimulation in nonobese and obese humans. J Clin Endocrinol Metab 2002, 87:3902-3910.

32. Ibrahim MM: Subcutaneous and visceral adipose tissue: structural and functional differences. Obes Rev 2010, 11:11-18.

33. Knott RM, Trayhurn P, Hesketh JE: Changes in insulin-receptor mRNA levels in skeletal muscle and brown adipose tissue of weanling rats during fasting and refeeding. Br J Nutr 1992, 68:583-592.

34. Desbuquois B, Tozzo E, Collinet M, Lopez S, Bortoli S, Amessou M: Regulation of insulin receptor expression and its gene. Ann Endocrinol 1993, 54:373-384.

35. Royer C, Lachuer J, Crouzoulon G, Roux J, Peyronnet J, Mamet J, Pequignot J, Dalmaz Y: Effects of gestational hypoxia on mRNA levels of
Glut3 and Glut4 transporters, hypoxia inducible factor- 1 and thyroid hormone receptors in developing rat brain. Brain Res 2000, 856:119-128.

36. Wajchenberg BL: Subcutaneous and visceral adipose tissue: their relation to the metabolic syndrome. Endocr Rev 2000, 21:697-738.

37. Giorgino F, Laviola L, Eriksson JW: Regional differences of insulin action in adipose tissue: insights from in vivo and in vitro studies. Acta Physiol scand 2005, 183:13-30.

38. Virtanen KA, Hallsten K, Parkkola R, Janatuinen T, Lonnqvist F, Viljanen T, Ronnemaa T, Knuuti J, Huupponen R, Lonnroth P, Nuutila P: Differential effects of rosiglitazone and metformin on adipose tissue distribution and glucose uptake in type 2 diabetic subjects. Diabetes 2003, 52:283-290.

39. Fernandez-Real JM, Menendez JA, Fruhbeck G, Moreno-Navarrete JM, Vazquez-Martin A, Ricart W: Serum HER-2 concentration is associated with insulin resistance and decreases after weight loss. Nutr Metab 2010, 7:20.

40. Tang BL: Inhibitors of neuronal regeneration: mediators and signaling mechanisms. Neurochem Int 2003, 42:189-203.

41. Dai SM, Shan ZZ, Nakamura H, Masuko-Hongo K, Kato T, Nishioka K, Yudoh K: Catabolic stress induces features of chondrocyte senescence through overexpression of caveolin 1: possible involvement of caveolin 1-induced down-regulation of articular chondrocytes in the pathogenesis of osteoarthritis. Arthritis Rheum 2006, 54:818-831.

42. Bartholomew JN, Galbiati F: Mapping of oxidative stress response elements of the caveolin-1 promoter. Methods Mol Biol 2010, 594:409-23.

43. Dasari A, Bartholomew JN, Volonte D, Galbiati F: Oxidative stress induces premature senescence by stimulating caveolin-1 gene transcription through p38 mitogen-activated protein kinase/Sp1-mediated activation of two GC-rich promoter elements. Cancer Res 2006, 66:10805-10814

44. Abdelmohsen K, Kuwano Y, Kim HH, Gorospe M: Posttranscriptional gene regulation by RNA-binding proteins during oxidative stress: implications for cellular senescence. Biol Chem 2008, 389:243-255.

45. Pavlides S, Tsirigos A, Vera I, Flomenberg N, Frank PG, Casimiro MC, Wang C, Fortina P, Addya S, Pestell RG, Martinez-Outschoorn UE, Sotgia F, Lisanti MP: Loss of stromal caveolin-1 leads to oxidative stress, mimics hypoxia and drives inflammation in the tumor microenvironment, conferring the "reverse Warburg effect": A transcriptional informatics analysis with validation. Cell Cycle 2010, 9:2201-2219.

46. Esposito K, Nappo F, Marfella R, Giugliano G, Giugliano F, Ciotola M, Quagliaro L, Ceriello A, Giugliano D: Inflammatory cytokine concentrations are acutely increased by hyperglycemia in humans: role of oxidative stress. Circulation 2002, 106:2067-2072.

47. Preis SR, Massaro JM, Robins SJ, Hoffmann U, Vasan RS, Irlbeck T, Meigs JB, Sutherland P, D'Agostino RB Sr, O'Donnell CJ, Fox CS: Abdominal subcutaneous and visceral adipose tissue and insulin resistance in the Framingham heart study. Obesity 2010, 18:2191-2198.

doi:10.1186/1476-511X-10-55

Cite this article as: Gómez-Ruiz et al:: High-fat diet feeding alters metabolic response to fasting/non fasting conditions. Effect on caveolin expression and insulin signalling. Lipids in Health and Disease 2011 10:55.

\section{Submit your next manuscript to BioMed Central and take full advantage of:}

- Convenient online submission

- Thorough peer review

- No space constraints or color figure charges

- Immediate publication on acceptance

- Inclusion in PubMed, CAS, Scopus and Google Scholar

- Research which is freely available for redistribution 